Department of Pathology, 1301 Catherine Road, 7520 MSRB I, Ann Arbor, MI 481095602, USA. Phone: 734.647.2921; Fax: 734.764.4308; E-mail: pward@umich.edu.

1. Pluddemann A, Mukhopadhyay S, Gordon S. Innate immunity to intracellular pathogens: macrophage receptors and responses to microbial entry. Immunol Rev. 2011;240(1):11-24.

2. Gustot T. Multiple organ failure in sepsis: prognosis and role of systemic inflammatory response. Curr Opin Crit Care. 2011;17(2):153-159.

3. Green S, Rothman A. Immunopathological mechanisms in dengue and dengue hemorrhagic fever. Curr Opin Infect Dis. 2006;19(5):429-436.

4. Bakker AB, Baker E, Sutherland GR, Phillips JH, Lanier LL. Myeloid DAP12-associating lectin (MDL)-1 is a cell surface receptor involved in the activation of myeloid cells. Proc Natl Acad Sci US A.
1999;96(17):9792-9796.

5. Chen ST, et al. CLEC5A is critical for dengue-virus-induced lethal disease. Nature. 2008; 453(7195):672-676.

6. Cheung R, et al. Activation of MDL-1 (CLEC5A) on immature myeloid cells triggers lethal shock in mice. J Clin Invest. 2011;121(11):4446-4461.

7. Eash KJ, Means JM, White DW, Link DC. CXCR4 is a key regulator of neutrophil release from the bone marrow under basal and stress granulopoiesis conditions. Blood. 2009;113(19):4711-4719.

8. Pillay J, et al. In vivo labeling with $2 \mathrm{H} 2 \mathrm{O}$ reveals a human neutrophil lifespan of 5.4 days. Blood. 2010; 116(4):625-627.

9. Simon HU. Neutrophil apoptosis pathways and their modifications in inflammation. Immunol Rev. 2003;193:101-110.

10. Ley K, Laudanna C, Cybulsky MI, Nourshargh S. Getting to the site of inflammation: the leukocyte adhesion cascade updated. Nat Rev Immunol. 2007; 7(9):678-689.
11. Lee H, Whitfeld PL, Mackay CR. Receptors for complement C5a. The importance of C5aR and the enigmatic role of C5L2. Immunol Cell Biol. 2008; 86(2):153-160.

12. Zhang H, Qin G, Liang G, Li J, Barrington RA, Liu DX. C5aR-mediated myocardial ischemia/reperfusion injury. Biochem Biophys Res Commun. 2007; 357(2):446-452

13. Hopken UE, Lu B, Gerard NP, Gerard C. The C5a chemoattractant receptor mediates mucosal defence to infection. Nature. 1996;383(6595):86-89.

14. Rittirsch D, et al. Functional roles for C5a receptors in sepsis. Nat Med. 2008;14(5):551-557.

15. Huber-Lang $M$, et al. Role of C5a in multiorgan failure during sepsis. JImmunol. 2001;166(2):1193-1199.

16. Ward PA. The harmful role of C5a on innate immunity in sepsis. J Innate Immun. 2010;2(5):439-445.

17. Bonder CS, Ajuebor MN, Zbytnuik LD, Kubes P, Swain MG. Essential role for neutrophil recruitment to the liver in concanavalin A-induced hepatitis. J Immunol. 2004;172(1):45-53.

\title{
The long and the short of aberrant ciliogenesis in Huntington disease
}

\author{
Jeh-Ping Liu and Scott O. Zeitlin
}

Department of Neuroscience, University of Virginia, Charlottesville, Virginia, USA.

\begin{abstract}
Huntington disease (HD) is a dominantly inherited neurodegenerative disorder that is caused by a mutant huntingtin $(H T T)$ gene encoding a version of the $\mathrm{Htt}$ protein with an expanded polyglutamine stretch. Although the HTT gene was discovered more than 18 years ago, the functions of normal $\mathrm{Htt}$ and the mechanisms by which mutant $\mathrm{Htt}$ causes disease are not well defined. In this issue of the JCI, Keryer et al. uncovered a novel function for normal $\mathrm{Htt}$ in ciliogenesis and report that mutant $\mathrm{Htt}$ causes hypermorphic ciliogenesis and ciliary dysfunction. These observations suggest that it is now critical to understand the extent to which ciliary dysfunction contributes to the different symptoms of HD and to determine whether therapeutic strategies designed to normalize ciliary function can ameliorate the disease.
\end{abstract}

Huntington disease (HD) is an autosomaldominant disorder caused by expansion of a CAG repeat in the first exon of the huntingtin (HTT) gene (1). This repeat encodes an expanded stretch of polyglutamine residues at the amino terminus of the $\mathrm{Htt}$ protein. HD is predominantly an adultonset disorder that is characterized by progressive neuronal cell death primarily in the striatum and deep layers of the cortex. Clinically, it is characterized by motor, cognitive, and neuropsychiatric abnormalities that cause a progressive loss of functional capacity and reduced life span (2). There are currently no effective treatments for

Conflict of interest: The authors have declared that no conflict of interest exists.

Citation for this article: J Clin Invest. 2011; 121(11):4237-4241. doi:10.1172/JCI60243. this devastating neurodegenerative disease. This stems largely from an incomplete understanding of the cellular and molecular mechanisms by which mutant Htt causes disease.

Evidence obtained from cell culture and animal model studies supports the hypothesis that the polyglutamine expansion in mutant Htt confers on the protein both a toxic gain of function and a partial loss of normal function (3). More than 100 Htt-interacting proteins have been identified, implicating $\mathrm{Htt}$ as a participant in a diverse array of cellular processes (4). One of the most predominant of these processes is microtubule-based transport of vesicles and organelles. The role of Htt in intracellular transport is mediated by its direct interaction with the dynein intermediate chain within the dynein microtubule motor complex (5) and by an indirect interaction with dynein via its association with a complex containing huntingtin-associated protein 1 (HAP1) and dynactin (6). In the presence of mutant Htt, dynein function is compromised, perturbing vesicle and organelle transport along microtubules.

In this issue of the JCI, Keryer and colleagues have linked the function of $\mathrm{Htt}$ in intracellular transport to ciliogenesis (7). As mutant Htt was found to cause hypermorphic ciliogenesis and ciliary dysfunction, it is possible that several symptoms of HD might be caused, at least in part, by ciliary dysfunction.

\section{Cilia and ciliopathies}

Primary cilia are single hair-like protrusions $1-5 \mu \mathrm{m}$ in length that are present on virtually all cells, including neurons and glia $(8,9)$. Primary cilia are nonmotile and have a microtubule skeleton consisting of nine microtubule pairs $(9+0$ axoneme), whereas motile secondary cilia have the same outer nine microtubule pairs, but include inner and outer dynein arms and a pair of central microtubules $(9+2$ axoneme) (Figure 1). Primary cilia in mammalian neurons are derived from a centriole within the centrosome and are located on the soma or proximal portion of the apical dendrite. They are thought to be involved 


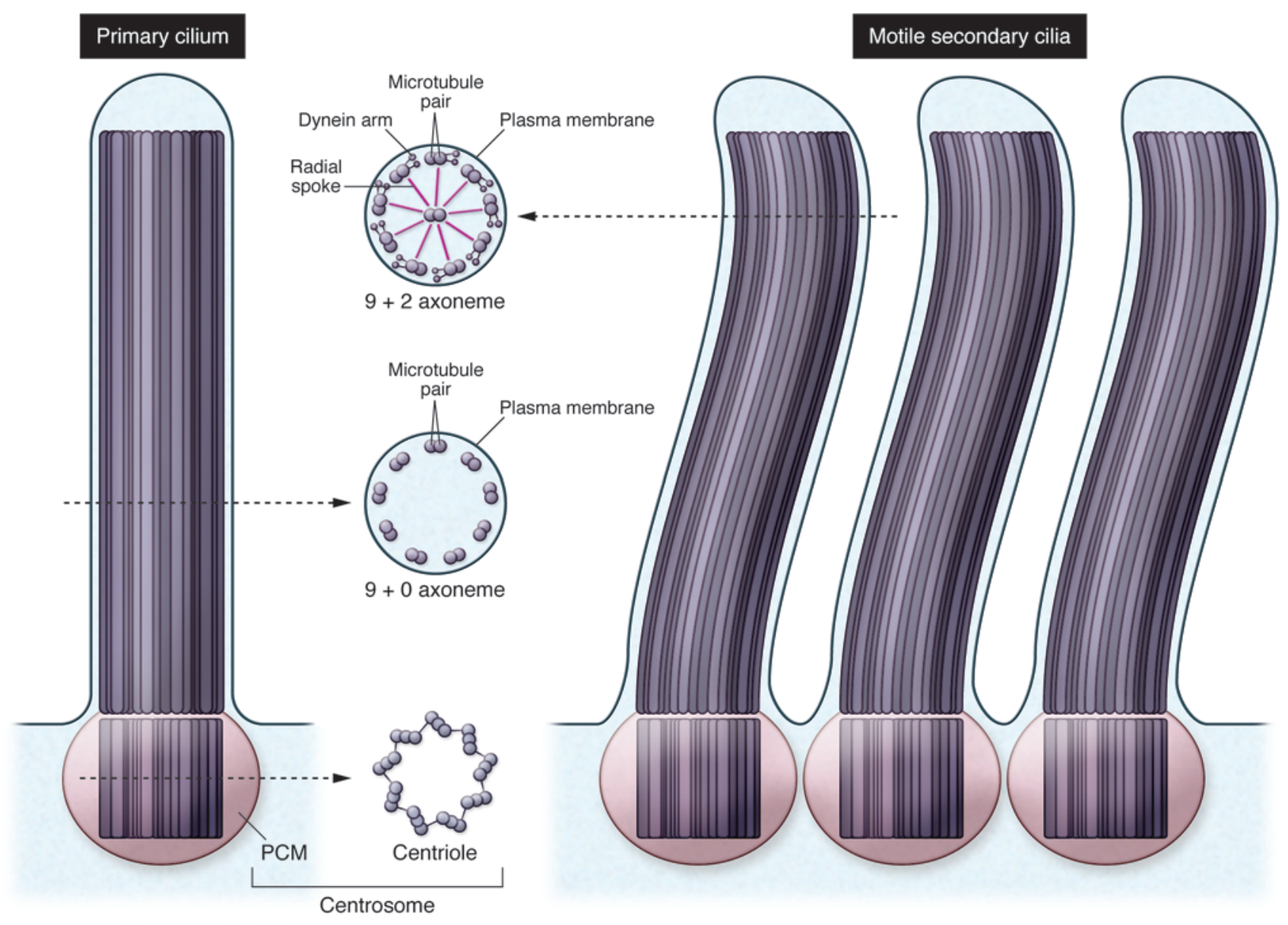

Figure 1

Structure of primary and motile secondary cilia. Primary and motile secondary cilia differ in the structure of their axoneme. Primary cilia have an axoneme composed of nine pairs of microtubules $(9+0$ axoneme), whereas the axoneme of motile secondary cilia also includes a central pair of microtubules $(9+2$ axoneme). Each of the nine outer pairs of microtubules in the axoneme of motile secondary cilia have inner and outer dynein arms that connect with an adjacent pair of outer microtubules and radial spokes connecting them with the central pair of microtubules. At the base of primary cilia is a basal body composed of a centrosome (centriole plus surrounding PCM). Motile secondary cilia also have basal bodies consisting of centrioles and PCM.

in sensing the extracellular environment and in signal transduction of the various sensory modalities. Motile secondary cilia in the brain (such as those found on ependymal cells lining the lateral ventricles) are critical for maintaining the flow of cerebrospinal fluid (CSF) through the ventricles and sensing the composition of the CSF.

There are several known genetic disorders that are caused by defects in primary cilium formation, structure, and function; these conditions are known as ciliopathies (8). Among these are a group of autosomal-recessive developmental disorders - including Joubert, Bardet-Biedel, and Meckel-Gruber syndromes - that include nervous system dysfunction among their many symptoms (Table 1). Abnormalities in primary cilia have also been observed recently in a mouse model of the adultonset neurodegenerative disorder familial amyotrophic lateral sclerosis (10). These observations, together with the data generated by Keryer et al. indicating that the mutant Htt protein underlying HD can drive ciliary dysfunction (7), suggest that aberrant cilia function might contribute to the pathogenesis of other adult-onset neurodegenerative disorders.

\section{A novel function for $\mathrm{Htt}$ in ciliogenesis}

Using both in vitro and in vivo systems, Keryer et al. provide evidence of a functional role for Htt in ciliogenesis (7). They found that Htt and HAP1 regulated ciliogenesis by transporting pericentriolar material 1 protein (PCM1) to the centro- some (Figure 2). In the absence of $\mathrm{Htt}$ or HAP1 expression, mislocalization of PCM1 reduced primary cilium formation in neurons and glia in culture and led to hypomorphic motile secondary cilia in ependymal cells. The latter contributed to hydrocephalus in an Htt loss-of-function mouse model.

In contrast to the hypomorphic ciliogenesis phenotype observed when normal Htt expression was reduced, Keryer et al. found that mutant Htt expression had the opposite effect on ciliogenesis (ref. 7 and Figure 2): excess PCM1 accumulated at the centrosome, the percentage of neurons and glia with primary cilia was increased in vitro, and ependymal cell cilia were lengthened in a mouse model of HD. In the model of HD, this phenotype led to 


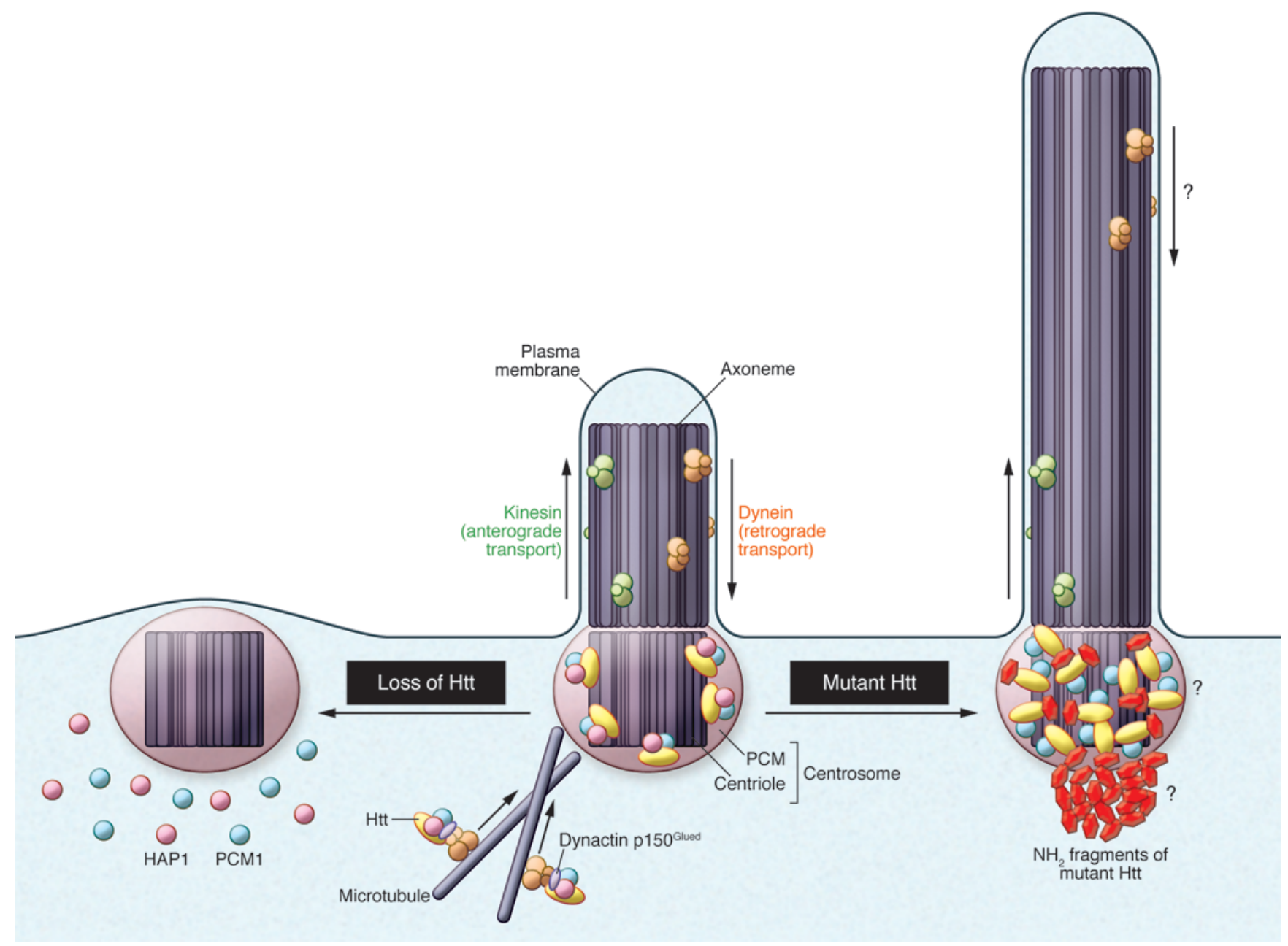

Figure 2

Mutant $\mathrm{Htt}$ and loss of normal Htt have opposing effects on ciliogenesis. Keryer and colleagues showed that mutant Htt drives increased ciliogenesis and the formation of hypermorphic primary cilia, whereas loss of $\mathrm{Htt}$ results in reduced ciliogenesis (7). These results suggest that normal $\mathrm{Htt}$ acts as a molecular scaffold in dynein/dynactin/HAP1-mediated transport of PCM1 to the centrosome. In the absence of Htt, dynein-mediated transport of PCM1 to the centrosome is impaired, and a primary cilium is not formed. In contrast, the presence of mutant Htt causes accumulation of PCM1 at the centrosome, increased ciliogenesis, and lengthening of the primary cilium. It is not yet known in HD whether dynein-mediated intraflagellar transport is affected, whether PCM1 is associated in a complex with mutant Htt and/or HAP1 at the centrosome, or whether aggresome formation can affect ciliary function.

abnormal CSF flow that affected neuroblast migration from the subventricular zone to the olfactory bulb. In addition, the authors observed an increase in PCM1 and $\mathrm{N}$-acetylated tubulin (a marker of cilia) immunoreactivity in postmortem brains of individuals with HD.

\section{Cilia dysfunction and HD symptoms}

The work of Keryer et al. (7) suggests that several symptoms of HD may be caused, at least in part, by dysfunctional primary and secondary cilia. Their data suggest that defects in ependymal motile cilia impair CSF flow, resulting in an abnormal rostral migratory stream (RMS). Adult neurogene- sis in the subventricular zone generates neuroblasts that migrate through the RMS into the olfactory bulb, where they differentiate into interneurons. Normal CSF circulation is required to orient the neuroblasts in the RMS, and the dysfunctional ependymal cilia observed by Keryer et al. in the mouse model of HD led to reduced CSF flow, affecting neuroblast migration (7). The authors speculate that a defective RMS may contribute to the olfactory deficits experienced by HD patients and that reduced CSF flow in HD may also affect brain homeostasis by altering the clearance of brain catabolites.

Neurons expressing olfactory receptors also have nonmotile secondary cilia that are important for normal function (11). In Bardet-Biedel syndrome, for example, defective cilia function in olfactory receptor neurons can cause anosmia in humans (12). Although not examined by Keryer and colleagues, it will be interesting to see whether hypertrophic cilia are observed in the olfactory epithelia of HD mouse models and patients, and if so, whether this structural abnormality affects olfactory receptor neuronal function.

The dentate gyrus region of the hippocampus contains adult neuronal stem and progenitor cells with primary cilia in both rodents and humans (13-15). Given that Keryer et al. identified defects in ependymal motile cilia and neuronal primary cilia (7), it 
Table 1

Autosomal-recessive ciliopathies associated with neuronal dysfunction

\begin{tabular}{lll}
$\begin{array}{l}\text { Disorder } \\
\text { Joubert syndrome }\end{array}$ & $\begin{array}{l}\text { Gene loci involved } \\
\text { AHI1, NPHP1, MKS3-MKS6, } \\
\text { ARL13B, INPP5E }\end{array}$ & $\begin{array}{l}\text { Symptoms with a neural basis } \\
\text { Developmental malformation of the cerebellum, cognitive deficits, behavioral deficits, } \\
\text { abnormal eye movements, brainstem malformations leading to episodic apnea or } \\
\text { hyperapnea, retinal dystrophy, variably penetrant cortical abnormalities }\end{array}$ \\
$\begin{array}{l}\text { Bardet-Biedel syndrome } \\
\text { Alström syndrome }\end{array}$ & $\begin{array}{l}\text { BBS1-BBS14 } \\
\text { Meckel-Gruber syndrome }\end{array}$ & $\begin{array}{l}\text { Retinal dystrophy, cognitive impairment, anosmia, obesity (hypothalamic dysfunction) } \\
\text { Retinal dystrophy, obesity (hypothalamic dysfunction), sensorineural hearing deficits }\end{array}$ \\
\hline
\end{tabular}

AMKS1 is also known as BBS13; MKS3 as TMEM67; MKS4 as CEP290 or BBS14; MKS5 as RPGRIP1L; MKS6 as CC2D2A.

will be important in future studies to determine whether adult neuronal stem cells in the brains of rodents and humans with HD have hypertrophic cilia and whether this phenotype correlates with deficits in adult neuronal stem cell function in HD. This may have direct relevance to cognitive dysfunction in HD, as it has previously been shown that adult hippocampal neurogenesis in rodents is involved in learning and memory (16).

The increased ciliogenesis in HD mouse model astrocytes observed by Keryer et al. (7) could also contribute to the deficits in astrocyte function that are observed in HD (17). Astrocytes have a critical role in maintaining neuronal homeostasis, and the increased ciliogenesis observed in HD may perturb the ability of astrocytes to sense and respond to changes in its environment, thus affecting neuronal survival.

\section{How does mutant Htt affect cilia function?}

Although aggregation of PCM1 in the choroid plexus and ependymal cell layer of the lateral ventricles was detected by Keryer et al. as early as 5 months of age in a mouse model of HD, abnormal cilia were only observed later, at 12 months of age (7). This phenotype appears to differ from the classic autosomal-recessive ciliopathies that exhibit early developmental abnormalities (Table 1), instead resembling the slow progression of degeneration that is characteristic of HD.

A neuropathological hallmark of HD is the accumulation of aggregated amino-terminal fragments of mutant Htt in the cytoplasm (neuropil inclusions) and nuclei (nuclear intranuclear inclusions). Small cytoplasmic Htt aggregates can be transported to the centrosome, where they coalesce to form an aggresome that can protect cells from the buildup of misfolded protein (18). The work of Keryer et al. (7) leads us to wonder whether the formation of the aggresome affects cilia function in the HD brain. The authors' observations also raise a number of other important questions: How does the aggregation of PCM1 affect ciliogenesis, ciliary maintenance, ciliary signaling, or some combination thereof? Are other ciliary cargos affected? Is transport along the axoneme (intraflagellar transport) also affected? These are critical questions, as the answers have direct relevance to the design of therapeutic strategies aimed at restoring the function of primary and motile cilia in HD patients.

In addition to perturbing HAP1 and dynein function (3), mutant Htt affects membrane trafficking by interfering with the activities of Rab8 and Rab11, two small GTPases that function in ciliogenesis (19-21). Mutant Htt expression disrupts both Rab8 and Rab11 activity by affecting normal localization of Rab8 on Golgi membranes (20) and by impairing guanine nucleotide exchange factor activity on Rab11, resulting in reduced recycling of cargo proteins (21). Expression of a constitutively active form of Rab11 in primary neurons from an HD mouse model ameliorates deficits in extracellular cysteine uptake by restoring cell surface levels of the glutamate transporter EAAC1 (22), and overexpression of Rab11 in a Drosophila model of HD rescues neurodegeneration and extends lifespan (23). Thus, it is possible that manipulating the activity and expression levels of HAP1, dynein, Rab8, and Rab11 might ameliorate at least some aspects of ciliary dysfunction in HD.

\section{Therapeutic perspectives for treating cilia dysfunction in HD}

The present study by Keryer and colleagues has uncovered a mechanism underlying HD pathogenesis that may provide a new therapeutic target for the disease (7). Although not all pathological events in HD can be explained by defective ciliogenesis and abnormal ciliary function, amelioration of those symptoms caused by abnormal primary and secondary ciliary function should improve the quality of life for patients with HD and may provide relief for symptoms that do not yet exhibit an obvious link to ciliary dysfunction. Moreover, as morphological and functional criteria for primary and secondary cilia already exist, the establishment of cell culture-based high-throughput screens for small molecules able to rescue the effects of mutant Htt on ciliogenesis and ciliary function should be feasible.

\section{Acknowledgments}

The authors thank Emily Andre for comments and suggestions. This work was supported in part by the NIH (NINDS NS043466) and by CHDI Foundation Inc.

Address correspondence to: Scott Zeitlin, Department of Neuroscience, University of Virginia School of Medicine, Box 801392, Charlottesville, Virginia 22908, USA. Phone: 434.924.5011; Fax: 434.982.4380; E-mail: soz4n@virginia.edu.

1. The Huntington's Disease Collaborative Research Group. A novel gene containing a trinucleotide repeat that is expanded and unstable on Huntington's disease chromosomes. Cell. 1993;72(6):971-983.

2. Hayden MR. Huntington's Chorea. New York, New York, USA: Springer-Verlag; 1981.

3. Zuccato C, Valenza M, Cattaneo E. Molecular mechanisms and potential therapeutical targets in Huntington's disease. Physiol Rev. 2010;90(3):905-981.

4. Harjes P, Wanker EE. The hunt for huntingtin function: interaction partners tell many different stories. Trends Biochem Sci. 2003;28(8):425-433.

5. Caviston JP, Zajac AL, Tokito M, Holzbaur EL. Huntingtin coordinates the dynein-mediated dynamic positioning of endosomes and lysosomes. Mol Biol Cell. 2011;22(4):478-492.

6. Rong J, Li SH, Li XJ. Regulation of intracellular HAP1 trafficking. J Neurosci Res. 2007;85(14):3025-3029.

7. Keryer G, et al. Ciliogenesis is regulated by a huntingtin-HAP1-PCM1 pathway and is altered in Huntington disease. J Clin Invest. 2011; 121(11):4372-4382.

8. Gerdes JM, Davis EE, Katsanis N. The vertebrate primary cilium in development, homeostasis, and disease. Cell. 2009;137(1):32-45.

9. Louvi A, Grove EA. Cilia in the CNS: the quiet organelle claims center stage. Neuron. 2011; 69(6):1046-1060.

10. Ma X, Peterson R, Turnbull J. Adenylyl Cyclase type 3, 
a marker of primary cilia, is reduced in primary cell culture and in lumbar spinal cord in situ in G93A SOD1 mice. BMC Neurosci. 2011;12:71.

11. Elsaesser R, Paysan J. The sense of smell, its signalling pathways, and the dichotomy of cilia and microvilli in olfactory sensory cells. BMC Neurosci. 2007;8(suppl 3):S1.

12. Kulaga HM, et al. Loss of BBS proteins causes anosmia in humans and defects in olfactory cilia structure and function in the mouse. Nature Genet. 2004;36(9):994-998.

13. Alvarez-Buylla A, Seri B, Doetsch F. Identification of neural stem cells in the adult vertebrate brain. Brain Res Bull. 2002;57(6):751-758.

14. Quinones-Hinojosa A, et al. Cellular composition and cytoarchitecture of the adult human subven- tricular zone: a niche of neural stem cells. J Comp Neurol. 2006;494(3):415-434.

15. Han Y-G, Alvarez-Buylla A. Role of primary cilia in brain development and cancer. Curr Opin Neurobiol. 2010;20(1):58-67

16. Deng W, Aimone JB, Gage FH. New neurons and new memories: how does adult hippocampal neurogenesis affect learning and memory? Nat Rev Neurosci. 2010;11(5):339-350.

17. Bradford J, et al. Mutant huntingtin in glial cells exacerbates neurological symptoms of Huntington disease mice. J Biol Chem. 2010;285(14):10653-10661.

18. Johnston JA, Ward CL, Kopito RR. Aggresomes: a cellular response to misfolded proteins. J Cell Biol. 1998;143(7):1883-1898

19. Knodler A, et al. Coordination of Rab8 and Rab11 in primary ciliogenesis. Proc Natl Acad Sci U S A. 2010; 107(14):6346-6351.

20. del Toro D, et al. Mutant huntingtin impairs postGolgi trafficking to lysosomes by delocalizing optineurin/Rab8 complex from the Golgi apparatus. Mol Biol Cell. 2009;20(5):1478-1492.

21. Li X, et al. Mutant huntingtin impairs vesicle formation from recycling endosomes by interfering with Rab11 activity. Mol Cell Biol. 2009;29(22):6106-6116.

22. Li X, et al. Aberrant Rab11-dependent trafficking of the neuronal glutamate transporter EAAC1 causes oxidative stress and cell death in Huntington's disease. J Neurosci. 2010;30(13):4552-4561.

23. Richards $P$, et al. Dendritic spine loss and neurodegeneration is rescued by Rab11 in models of Huntington's disease. Cell Death Differ. 2011;18(2):191-200.

\title{
Are maternal antiplatelet antibodies a prothrombotic condition leading to miscarriage?
}

\author{
Alvin H. Schmaier \\ Case Western Reserve University, Cleveland, Ohio, USA.
}

\begin{abstract}
Fetal and neonatal alloimmune thrombocytopenia (FNAIT) is a condition characterized by thrombocytopenia in the newborn. If severe, the thrombocytopenia can lead to intracranial hemorrhage. FNAIT arises when maternal antibodies specific for platelet antigens, most commonly $\beta 3$ integrin, cross the placenta and destroy fetal platelets. Surprisingly, few cases of FNAIT are associated with antibodies specific for the platelet antigen GPIb $\alpha$, which is a common target in patients with immune thrombocytopenia. In this issue of the JCI, Li et al. have identified a potential reason for this - they find that in the majority of pregnant mice, anti-GPIb $\alpha$ antibodies enhance platelet activation and accelerate thrombus formation in the placenta and that this leads to miscarriage.
\end{abstract}

Our parents remind us that we should not forget our roots. Hematologists should not forget that the discipline over the last 100 years partly arose to prevent the large number of hemorrhagic deaths associated with labor and delivery (they occurred in $0.25 \%$ of deliveries in 1900 and $0.004 \%$ of deliveries in 2005) (1). Some success has been achieved, for example, in the development of approaches to detect and treat rhesus D hemolytic disease of the newborn: the indirect Coombs test (indirect antiglobulin test), which is used to determine whether a mother has been sensitized by red cell antigens (e.g., the rhesus D antigen $[\mathrm{RhD}]$ ) from a previous pregnancy (2); and the first immunoglobulin therapy, involving the administration of RhD-specific

Conflict of interest: Alvin H. Schmaier receives research support from CSL Behring.

Citation for this article: J Clin Invest. 2011; 121(11):4241-4243. doi:10.1172/JCI60749. antibody to RhD-negative mothers who have been exposed to fetal RhD blood antigens. However, many hematological conditions continue to pose serious problems for individuals who are pregnant, including preeclampsia with hypertension and thrombocytopenia and fetal and neonatal alloimmune thrombocytopenia (FNAIT; also known as neonatal alloimmune thrombocytopenia [NAIT], fetal alloimmune thrombocytopenia [FAIT], or fetal neonatal immune thrombocytopenia [FNIT]).

\section{Maternal antiplatelet antibodies in pregnancy} ing in individuals of European descent in $0.06 \%-0.1 \%$ of live births (3). It is characterized by thrombocytopenia shortly after birth. Twenty percent of women with known prior immune thrombocytopenia will deliver a fetus with a platelet count of less than $50,000 / \mu \mathrm{l}(4)$. Although the thrombocyto-
FNAIT is a relatively rare condition, aris- penia is often mild and the affected neonate remains largely asymptomatic, cases of severe thrombocytopenia carry a high risk of intracranial hemorrhage (5). In fetuses with a platelet count of less than $20,000 / \mu 1$, the incidence of intracerebral hemorrhage is $10 \%-20 \%$, which may lead to neurological impairment or death (5).

FNAIT arises when maternal antibodies specific for platelet antigens cross the placenta and destroy fetal platelets. The maternal antibodies may arise during pregnancy, if fetal platelet antigens are recognized by the maternal immune system as nonself (allogeneic), or be already present in a mother with immune thrombocytopenia. In mothers with immune thrombocytopenia, it is also possible that the situation is not passive, since these individuals may be more sensitive to developing antibodies specific for allogeneic fetal platelet antigens.

Most commonly (in $79 \%-85 \%$ of cases of FNAIT), the pathogenic antibodies are specific for the HPA-1a epitope of $\beta 3$ integrin, a component of one of the major glycoproteins on the surface of platelets $(3,6,7)$. Other targets of the pathogenic maternal alloantibodies include the HPA5 b epitope of GPIa (also known as integrin a2) (in 7\%-10\% of cases) and both HPA-1a and HPA- $5 \mathrm{~b}$ (in $2 \%-7 \%$ of cases) $(3,6,7)$. It is of interest that few cases of FNAIT are associated with antibodies specific for epitopes of GPIb $\alpha$ (HPA-2a/b), a component of another major glycoprotein on the surface 